\title{
Analyse et enseignement des discours sur l'eau en FOS
}

\author{
Mangiante, Jean-Marc
}

Université d’Artois - Grammatica, jmarc.mangiante@univ-artois.fr

\section{Resumen}

Este artículo es parte del eje del coloquio dedicado al FOS y tiene como objetivo presentar los diferentes discursos sobre el tema del agua, procedentes de los corpus creados en el grupo de investigación Grammatica de la Universidad de Artois, con el fin de analizar el uso de la lengua en ámbitos profesionales y académicos. Este artículo permitirá identificar las aportaciones de las herramientas digitales del discurso y del análisis didáctico del FOS tales como los repositorios de competencias en la lengua profesional y académica diseñados para la formación en FOS y en FOU, alrededor del tema transversal del agua, tanto en el estudio del léxico como en el de la sintaxis.

Palabras clave : Análisis del discurso ; FOS ; herramientas digitales ; repositorios ; competencias.

\section{Résumé}

Cet article s'inscrit dans l'axe du colloque consacré au FOS et se propose de présenter les différents discours autour de la thématique de l'eau, issus des corpus constitués au sein du laboratoire Grammatica de l'université d'Artois en vue d'analyser la pratique de la langue en milieux professionnels et universitaires. Il sera l'occasion de dégager les apports des outils numériques d'analyse de discours et didactique du FOS comme les référentiels de compétences en langue professionnelle et universitaire conçus dans la perspective de formations en FOS et en FOU, autour de la thématique transversale de l'eau, déclinée dans l'étude du lexique comme de la syntaxe.

Mots-clés : Analyse de discours ; FOS ; outils numériques ; référentiels ; compétences.

\begin{abstract}
This article is in line with the symposium devoted to the FOS and proposes to present the different discourses around the theme of water, resulting from the corpus constituted in the laboratory Grammatica of the University of Artois, to analyze the practice of language in professional and academic circles. It will be an opportunity to highlight the contributions of the digital tools of analysis of discourse and didactics of the FOS as the references of competences in professional and academic language created in the aim of trainings in FOS and in FOU, about the transversal theme of the Water, declined in the study of the lexicon as of the syntax.
\end{abstract}

Keywords : Discourse analysis ; FOS ; numerical tools ; repositories ; competences. 


\section{Introduction}

Cet article s'inscrit dans la thématique de la didactique du Français sur Objectif Spécifique (FOS) appliquée aussi bien au domaine professionnel de l'apprentissage du français qu'au domaine universitaire pour la formation des étudiants allophones. Cette démarche d'ingénierie de formation (Mangiante et Parpette, 2004 et 2011) intègre une étape essentielle d'analyse des discours professionnels ou universitaires collectés sur le terrain dans la perspective de leur exploitation pédagogique en cours de langue. C'est dans ce cadre que la constitution de corpus authentiques contribue à améliorer la connaissance de ces secteurs d'activité souvent étrangers à l'enseignant de langue.

L'article se propose de présenter les différents discours autour de la thématique de l'eau, issus des corpus constitués au sein du laboratoire Grammatica de l'université d'Artois en vue d'analyser la pratique de la langue en milieux professionnels et universitaires.

Elle sera l'occasion de dégager les apports des outils numériques d'analyse de discours et didactique du FOS comme les référentiels de compétences en langue professionnelle et universitaire conçus dans la perspective de formations en FOS, autour de la thématique transversale de l'eau, déclinée dans l'étude du lexique comme de la syntaxe.

Après une présentation des référentiels de compétences en langue appliqués au monde professionnel, conçus et utilisés par Grammatica, nous essaierons de dégager la place de l'eau dans les tâches professionnelles analysées dans les référentiels ainsi que dans les discours sur et autour de l'eau. Le recours aux corpus professionnels numérisés conduit ainsi les enseignants de langue à didactiser des discours à partir d'analyses lexicales et syntaxiques qui s'appuient sur des logiciels d'étude fréquentielle du discours (lexico 3, Tropes...).

Nous en déduirons l'importance de la constitution des référentiels et de l'analyse des corpus dans la conception de programmes de formation en FOS.

L'objectif est de présenter les différents discours autour de la thématique de l'eau, issus des corpus constitués au sein du laboratoire Grammatica de l'université d'Artois et de mettre en évidence les apports des outils numériques d'analyse de discours et didactique du FOS comme les référentiels de compétences en langue professionnelle conçus dans la perspective de formations en FOS et en FOU, autour de la thématique transversale de l'eau.

\section{Référentiels et corpus en FOS}

De nombreux discours professionnels oraux et écrits ont été collectés (enregistrements audio et vidéo, recueil de textes écrits...) dans le cadre de collaborations entre le laboratoire Grammatica et des entreprises du BTP, directement sur des chantiers, ainsi qu'avec le lycée hôtelier Marguerite Yourcenar de Beuvry pour le secteur de l'hôtellerie - restauration, selon une démarche de référentialisation (Mangiante, 2011). Cette dernière consiste d'abord à relever, sur le terrain, les situations et tâches professionnelles en vue d'en dégager « la part langagière du travail » (Boutet, 2001). Les discours professionnels collectés ensuite (enregistrements audio ou vidéo des échanges, recueil des documents écrits, lettres, tableaux, grilles, bon de commandes...), sont analysés à partir de logiciels d'analyse de discours (Tropes, Lexico 3...), pour mettre en évidence leurs caractéristiques linguistiques (lexique de spécialité, récurrences syntaxiques, occurrences, types discursifs dominants, relations grammaticales fréquentes...). Dans un second temps, les résultats des analyses permettent de dégager les compétences langagières associées aux différentes tâches professionnelles observées afin d'établir un «référent ", c'est-à-dire un recueil et un classement des compétences langagières nécessaires à un accomplissement idéal (dans le sens de la réalisation d'une performance efficace) des tâches professionnelles visées (Hadji, 2000).

L'ensemble des données analysées et des compétences dégagées aboutit à la construction de référentiels de compétences langagières en milieu professionnel :

- Pour les métiers du BTP.

- $\quad$ Le secteur de l'hôtellerie - restauration.

- Le secteur de l’hygiène - propreté (en cours) ...

- Le secteur de l’Aide à la Personne (en projet). 
Ces référentiels constituent ainsi des outils destinés aux centres de formation en langue et aux laboratoires de recherche pour leur permettre d'analyser des corpus collectés sur le terrain professionnel, de concevoir des formations et des évaluations en FOS et pour former les enseignants en FOS. Ils complètent et prolongent le Cadre européen commun de Référence pour les Langues (CECR-L) dont les situations de communication analysées pour la conception de ce référentiel ne portent pas sur le milieu professionnel.

Les figures suivantes sont issues des référentiels de compétences mis en ligne pour les chercheurs de Grammatica :
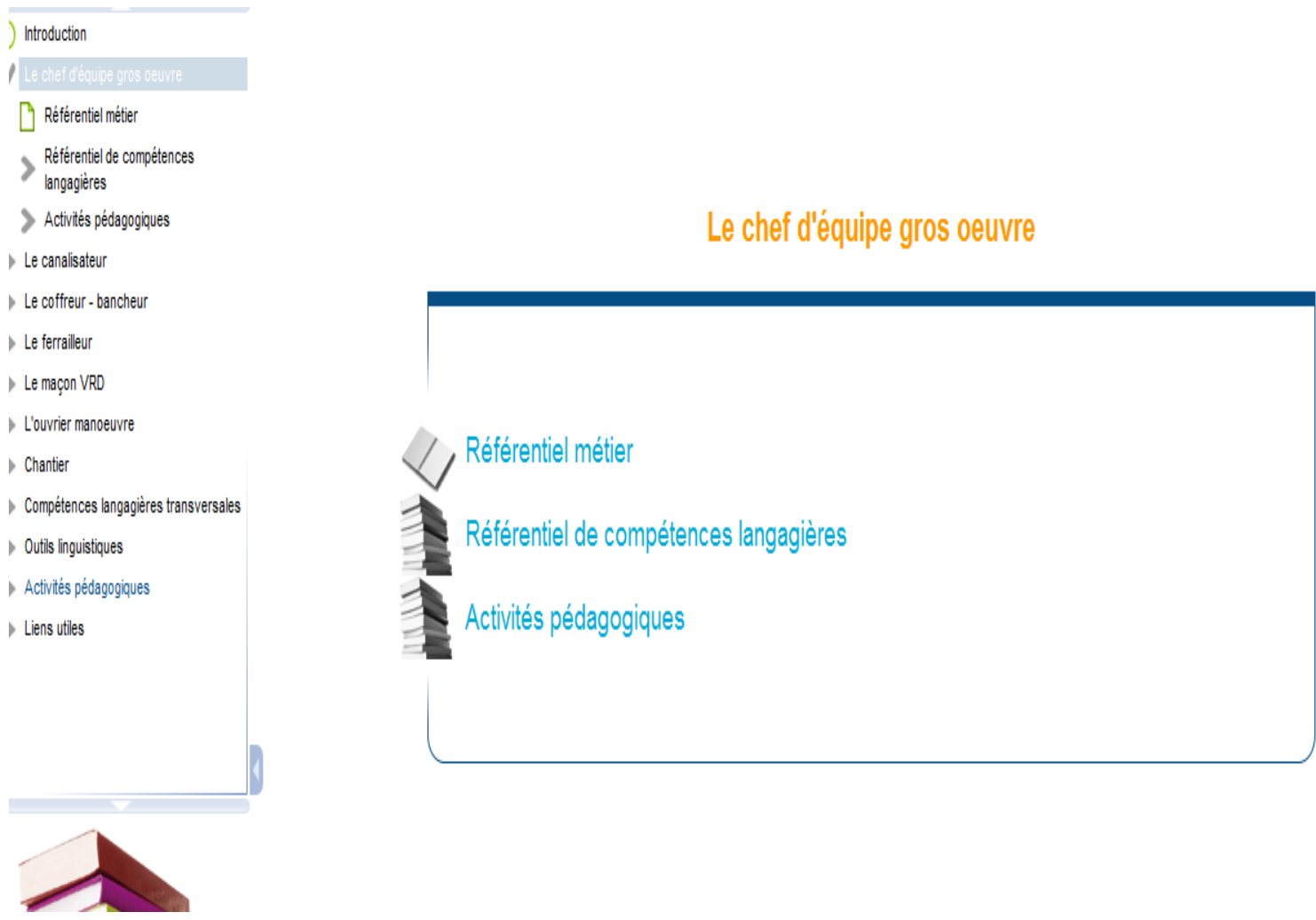

Fig. 1. Extrait du référentiel BTP, menu par métiers. 


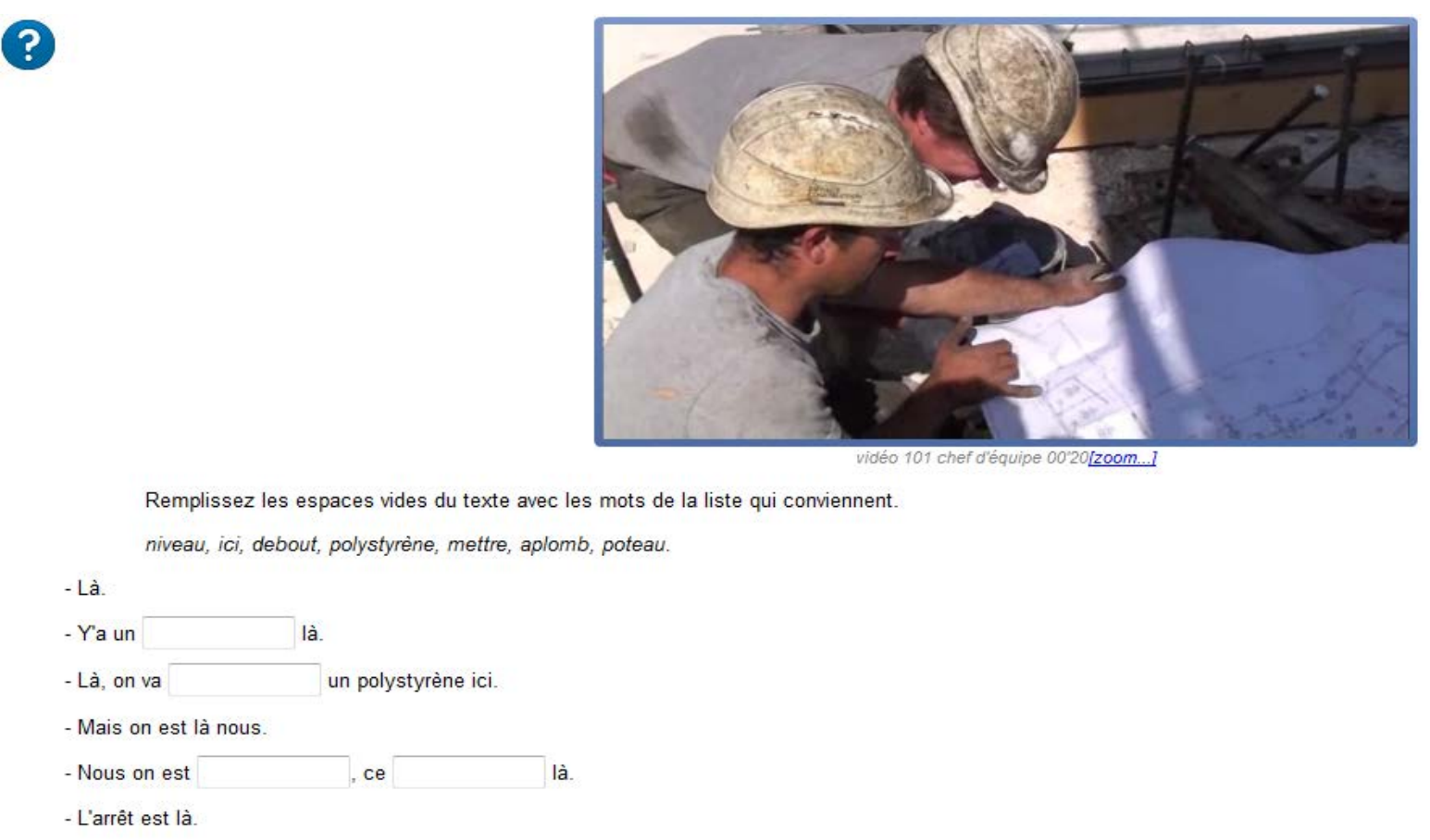

Fig. 2. Référentiel BTP, exemple d'activité pédagogique en ligne.

Outre le lexique spécialisé du BTP issu des enregistrements vidéo réalisés sur le chantier, le référentiel comporte des listes de compétences langagières, les structures grammaticales récurrentes et des exemples d'activités pédagogiques à intégrer dans un programme de FOS.

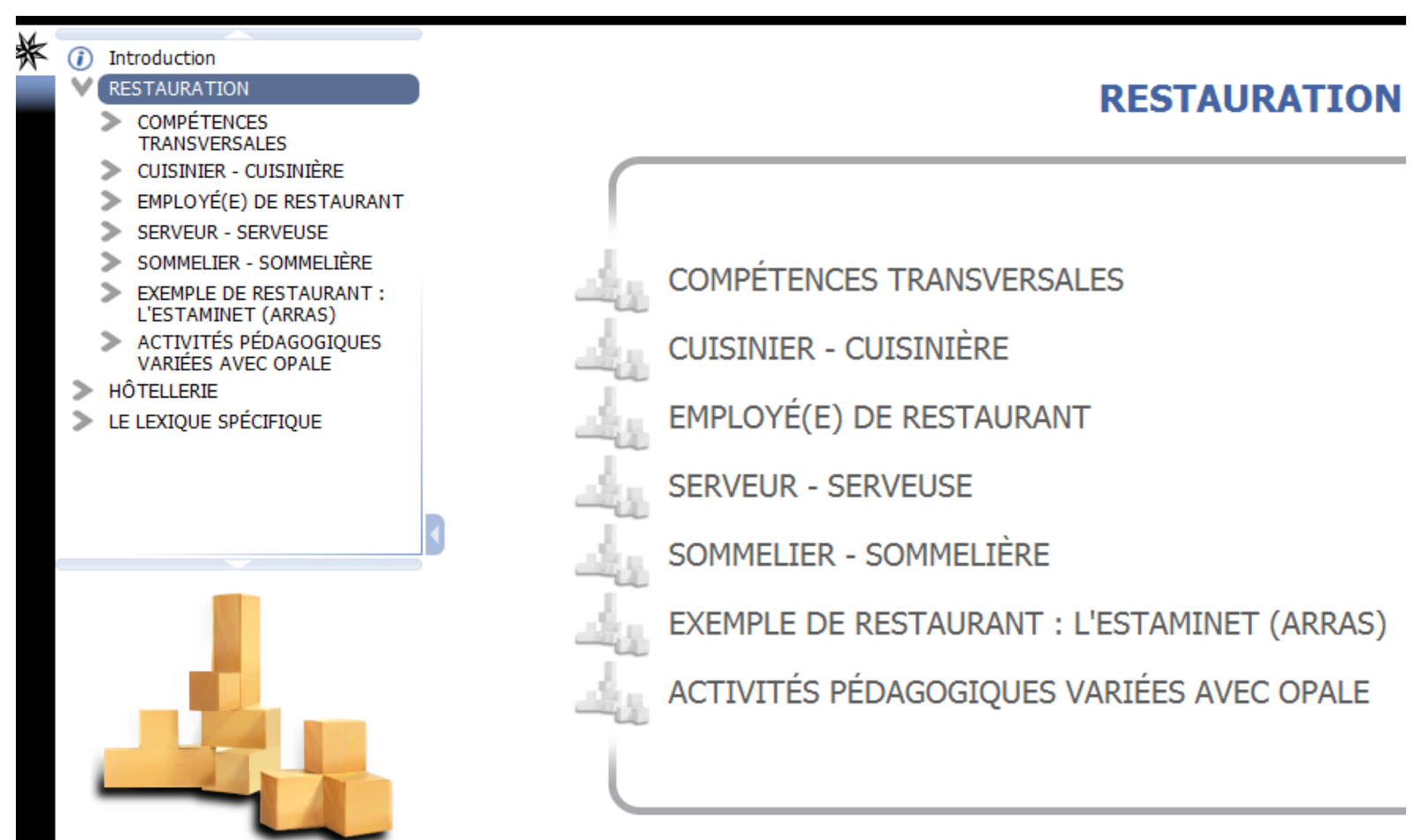

Fig. 3. Extrait du référentiel de compétences pour les métiers de la restauration. 


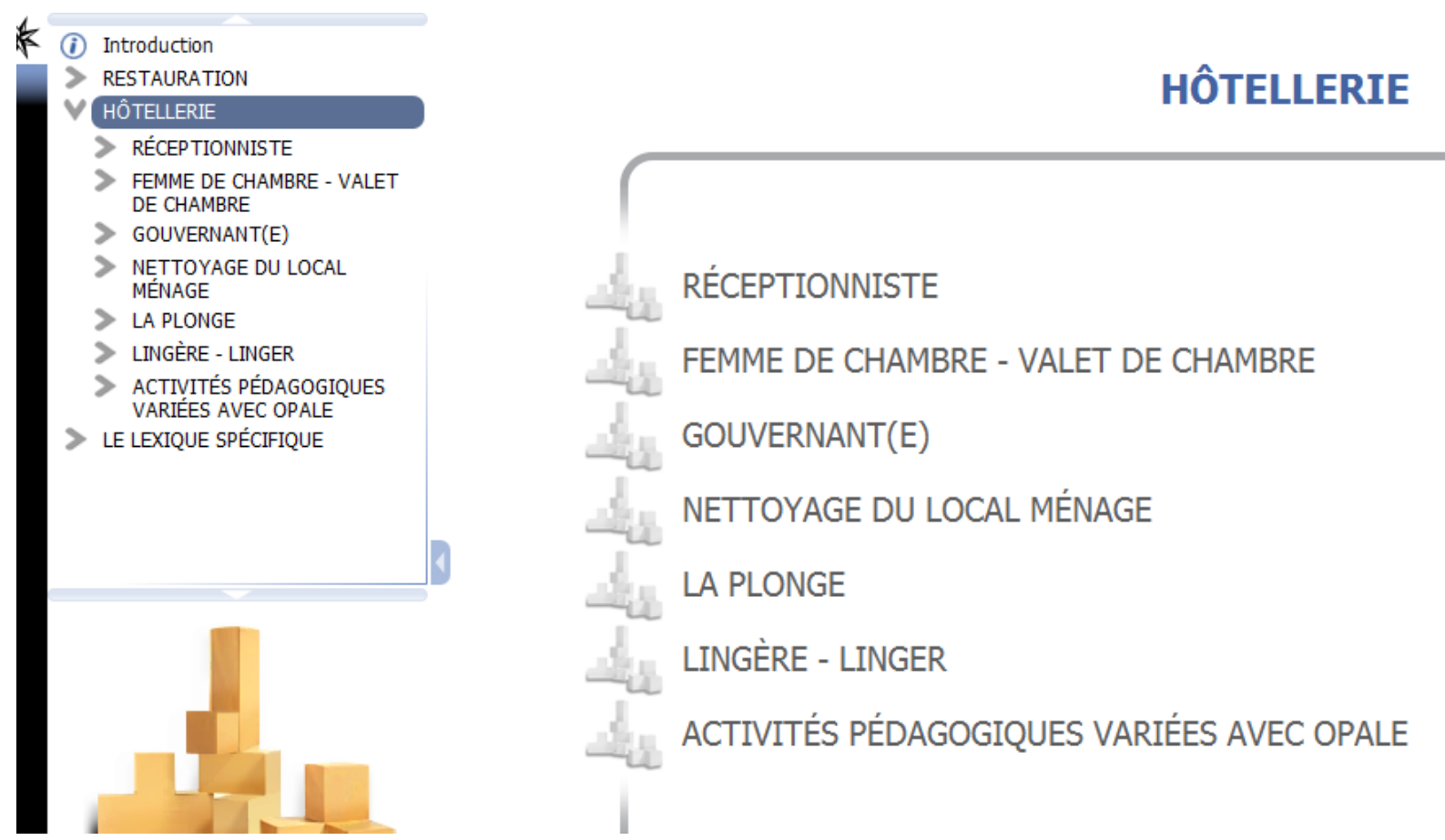

Fig. 4. Extrait du référentiel de compétences pour les métiers de l’hôtellerie.

\section{Quelques pistes d'analyse des discours professionnels}

L'analyse des discours professionnels issus des tâches de travail enregistrées sur le terrain révèle l'interdépendance du « langage au travail et du contexte d'action et l'interdépendance entre les activités verbales et non verbales, entre les activités verbales et les outils... » (Boutet et Maingueneau, 2005). Au sein de la tâche professionnelle, les opérateurs alternent gestes et paroles, supports écrits et objets ou matière...

Il se dégage ainsi une multicanalité des situations de travail : «va-et-vient entre écrit, oral, image... » et des activités de « transcodage ou transfert d'information d'un support à l'autre, de l'oral à l'écrit... » (Mourlhon-Dallies, 2008) qui seront autant de pistes à privilégier dans l'apprentissage du FOS pour faire comprendre aux apprenants les mécanismes langagiers à l'œuvre dans la communication en français sur le lieu de travail.

L'analyse met également en évidence les trois dimensions du langage en milieu professionnel (Green, 1997, repris par Grünhage-Monetti, 2003) :

- La dimension opérationnelle (ou fonctionnelle): la langue permet l'accomplissement de l'action, elle l'accompagne...

- La dimension culturelle : la langue révèle une pratique socio-culturelle liée au domaine professionnel et à la culture nationale (voire régionale)

- La dimension critique : la langue produit une réflexivité sur l'action et ses résultats, elle explique et corrige l'action au travail.

Ainsi l'analyse met en lumière les liens étroits entre langue et action, à l'origine de l'approche actionnelle développée par le CECR-L en illustrant le principe qu'il « n’y a de compétence que de compétence en acte. » (Le Boterf, 2000).

Les discours professionnels collectés peuvent également être réunis en "genres professionnels » partageant des caractéristiques linguistiques communes liées aux différents contextes professionnels dont ils sont issus. Ils en renouvèlent la définition à la lumière de leurs liens avec l'action au travail : « le concept de genre tel qu'élargi par les récents travaux en analyse des discours, en linguistique textuelle... peut constituer un excellent outil conceptuel afin de penser le langage et l'action dans leur imbrication » (Richer, 2008 : 117). 
Ces genres professionnels constituent des « entités langagières, culturelles et socio-historiques (Beacco, 2004) également définis ainsi : «... tout membre d’une communauté est confronté à un univers de textes "déjà là", univers organisé en "genres" empiriques et historiques, c'est-à-dire en formes d’organisation concrètes qui se modifient avec le temps. » (Bronckart, 2004).

L'enseignant - concepteur de formations en FOS doit nécessairement comprendre les différents paramètres contextuels de l'action au travail. Il doit maîtriser préalablement le protocole actionnel des tâches professionnelles (ou script d'action) qui établit la progression cohérente des actes et gestes professionnels conduisant au résultat de la tâche, et comprendre le statut des (inter)actants (liens hiérarchiques, positionnement dans l'entreprise ou l'institution...) et les objectifs de la tâche.

La langue au travail relève du protocole opérationnel de la tâche professionnelle à accomplir et de son interprétation par le locuteur : « travail prescrit codifié (protocolisé), travail réel et travail interprété » (Bronckart, 2004).

Concrètement, le protocole opérationnel induit des liens particuliers entre langue et action et des discours imbriqués dans l'action (Mangiante et Meneses, 2016) :

- Discours d'accompagnement de l'action,

- Discours de prescription de l'action (injonction),

- Discours d'explication / argumentation avant, pendant ou après l'action

- Des discours d'évaluation / modification de l'action

- Des discours de déclenchement /suspension / arrêt de l'action...

\subsection{Une piste d'analyse et d'enseignement : présence de l'eau dans les tâches et discours professionnels}

Les cadres conceptuel et contextuel de l'analyse des discours professionnels étant posés, nous pouvons dégager la présence du champ sémantique de l'eau au sein des corpus issus des référentiels. Cet exemple d'étude permet d’illustrer le rôle et l'utilisation des outils numériques d'analyse de discours et des référentiels dans une perspective didactique en FOS.

Les analyses révèlent quatre principales récurrences linguistiques de l’eau au sein des corpus :

L'eau est d'abord associée directement à la tâche professionnelle comme instrument, ingrédient, composante ou accompagnement.

\section{Exemple $n^{\circ} 1$ : transcription de l'enregistrement 138 (référentiel restauration)}

- « ...individuellement. Les mille-feuilles on va les faire ronds comme ceci. D’accord ? [...] Le montage se fera par couches successives de feuilletage, crème pâtissière, feuilletage. Et au moment de glacer au fondant on va enlever tout ça, d'accord, glacer au fondant, décorer de chocolat et chemiser d'amandes effilées torréfiées. On va faire des jolis mille-feuilles, des jolis savarins [...].

- On va travailler pour 8 chacun donc ça fera une détrempe à 250 grammes de farine, d’accord, 125 grammes d'eau, alors la détrempe on va faire la démonstration si vous voulez bien, on revient dessus. Donc, ce qu'on verra : le feuilletage, la pâte à savarin, la pâte à frire. Tout simplement. » [...].

Dans cet échange l’eau est associée à la tâche de réalisation d’une pâte feuilletée pour un mille-feuilles.

L’eau est également associée à l’action au travail : nettoyage, hygiène, propreté.

Exemple $n^{\circ} 2$ : transcription de l'enregistrement n59 (référentiel hygiène et propreté)

- « Fais pas de bêtises je mets la sauce. En fait y’a plein de mousse. Non mais ça c’est pas le bon tuyau Erina pour l'instant j'ai vidé l'eau propre. 
- bah non c'est l'eau sale que tu as vidé. Bah non c'est l'eau propre. Bah je te dis que c'est l'eau sale que t'as vidé. Regarde y'a rien qui coule. Ah si c'est bon. Erina t'as nettoyé le filtre parce que la dernière fois je me suis fait engueuler. Le filtre ? Bah y’a trop de mousse ici là. Tout y partira pas hein. Vous avez vidé le réservoir d'eau propre ? Non pas encore. On commence toujours par quoi ? Du plus propre au plus sale. Parce qu’elle s’est trompé de tuyau... »

Elle relève aussi de la relation de travail : service en salle ou commande à l’hôtel par exemple. Enfin elle est associée à des pratiques interculturelles (professionnelles et d'enseignement) : place de l'eau au restaurant par exemple différente selon les cultures.

\section{Exemple $n^{\circ} 3$ : Transcription enregistrement 53 (référentiel hôtellerie)}

- «Alors, le petit-déjeuner comprend une boisson chaude au choix, un jus de fruit, une corbeille du boulanger qui comprend croissant, petit pain, baguette, accompagné de beurre, confiture, miel, chocolat. Vous allez prendre quoi en boisson chaude?

- Donc, café au lait.

- Café au lait. Pour une personne, le petit-déjeuner ?

$-\quad[\ldots]$

- $\quad$ Il vous faut encore autre chose ?

- Non, ce sera tout.

- Alors, je récapitule : vous avez demandé un café noir avec un jus d’orange, avec une salade de fruits et un yaourt, et...

- $\quad$ Ah, j’ai oublié : une demi-bouteille d'eau, s’il vous plaît.

- Une demi-bouteille d'eau ? C’est noté aussi.

- Merci. »

\subsection{L'eau au sein de la tâche professionnelle}

La tâche professionnelle peut être définie comme le «but que l'utilisateur (ou opérateur) vise à atteindre assorti d'une procédure (ou plan ou script d'action) qui décrit les moyens pour atteindre ce but » (Normand, 1992). La procédure est un composant exécutif de la tâche : ensemble d’opérations organisé temporelles et structurelles.

La tâche se compose donc d'une série organisée d’opérations, selon une procédure connue à l'avance mais dont l'application peut varier selon les opérateurs, les entreprises (prescripteurs de la tâche), les cultures professionnelles...

Elle comprend également: la posture de l'opérateur, la routine dans l'exécution, un protocole culturel dans l'accomplissement...

Dans ce cadre l'eau peut apparaître comme objet de la tâche (action sur l'eau, modification de son état) : chauffer de l'eau, mélanger de l'eau avec, congeler de l'eau, faire bouillir l'eau...

L'eau peut aussi constituer une étape de la procédure : ajouter de l'eau, retirer de l'eau, verser de l'eau, renverser de l'eau, diluer un produit avec de l'eau...

L'eau peut être aussi considérée comme un élément de la tâche effective / perçue par l'opérateur et éventuellement corrigée : « tu as mis trop d'eau, c’est trop liquide, il n’y a pas assez d’eau, c’est épais... Tu as vidé l’eau ? De l'eau propre à l'eau sale...».

Cette présence de l'eau dans ces différentes fonctions révèle les régulations qui déterminent les genres professionnels analysés (ici les enregistrements de dialogues sur le lieu de travail au sein de tâches professionnels réalisées en même temps) : 
- Une régulation actionnelle: les énoncés accompagnent la progression des opérations constituant la tâche professionnelle ( «le montage se fera par couches successives de feuilletage, crème pâtissière, feuilletage »;

- Une régulation culturelle : les discours révèlent un ensemble de codes culturels régissant la relation entre les interactants («alors, le petit-déjeuner comprend une boisson chaude au choix, un jus de fruit, une corbeille du boulanger»);

- Une régulation communicationnelle et énonciative : les discours répondent à des règles liées à différents paramètres énonciatifs : cadre, moment, statut des interactants, enjeu de la communication... («on commence toujours par quoi ? Du plus propre au plus sale. Parce qu'elle s'est trompé de tuyau »).

\section{Ressources lexicales des référentiels de compétences : domaine et cotexte des occurrences de l'eau}

Le lexique spécialisé a été dégagé des différents référentiels professionnels afin de réaliser des analyses linguistiques spécifiques à l'aide de différents outils numériques.

Les ressources lexicales des référentiels constituent un atout indéniable dans la mesure où, pour le français, les corpus d’oral transcrit sont rares. L’étude du « parlé » présente un déficit au niveau des études linguistiques et les transcriptions sont très couteuses : une transcription fine peut demander jusqu’à 40 ou 60 fois le temps d'enregistrement (Habert, 2005).

L’analyse des corpus lexicaux ont suivi, au sein de Grammatica, le déroulement suivant :

- $\quad$ Enregistrements (transformation du format : mp4)

- Transcriptions (format word, txt, logiciel ELAN)

- Traitement du corpus par « domaine » = sous corpus professionnels

- $\quad$ La constitution de ressources avec l'instrument Unitex (concordanciers, dictionnaires électroniques, etc.).

L'étude des corpus repose sur la notion de « domaine » (corpus et sous corpus professionnels) et celle de cotexte défini comme « l’environnement immédiat linguistique » (C. Kerbrat-Orecchioni, 2012).

En effet, le texte isolé n’a pas plus d'existence que le mot ou la phrase isolés : pour être produit et compris, il doit être rapporté à un genre et à un discours, et par là à un type de pratique sociale. (Mangiante et Meneses, 2016).

Le corpus est d’ailleurs défini comme « un regroupement structuré de textes intégraux, documentés, éventuellement enrichis par des étiquetages, et rassemblés : (i) de manière théorique réflexive en tenant compte des discours et des genres, et (ii) de manière pratique en vue d’une gamme d’applications. » (F. Rastier, 2015).

L'analyse lexicale permet de dégager les mots connus et inconnus (verbes appropriés : prendre + vocabulaire), les collocations (verbes appropriés : nettoyer, verser, diluer, mélanger, chauffer, bouillir, écoper, pomper...), les locutions :

- $\quad$ locutions nominales eau plate, eau vive, eau morte, eau propre, eau sale

- $\quad$ locutions verbales, adverbiales, etc.

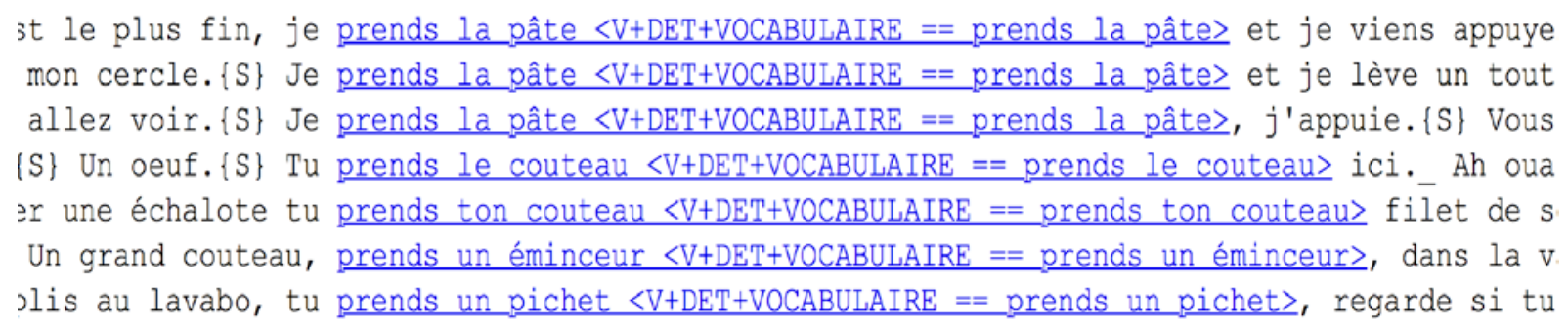

Fig. 5. Extrait de l'analyse du lexique de la restauration (référentiel restauration). 
On nettoie le plan de travail ! SS\} Faut du papier là!\{S\} Toujours à la poubelle! is préparer le plan de travail.\{S\} Alors.\{S\}.. On mettra un peu de verdure, pour 1 a colle sur le plan de travail qu'est ce qu'on va faire ?\{S\} Qu'est-ce que je fais Fleurer notre plan de travail. \{S\} Donc fleurer, je rappelle, ce n'est pas...ça.\{S rons sur votre plan de travail, chacun. $\{\mathrm{S}\}$ Tiens donnes-en deux à truc._C'est pas ès, tout votre plan de travail va coller.\{S\} L'idéal aussi c'est de travailler sur lains, ou votre plan de travail.\{S\} Je n'ai pas de torchons.\{S\} Ça. </transcriptior on nettoie son plan de travail, en attendant que ça cuise.\{S\} On jette les bains-m on nettoie son plan de travail avec du papier, OK?! </transcription> </transcripti légèrement son plan de travail....et on graisse...le dos de la cuillère.\{S\} Et à $c$

Fig. 6. Extrait de l'analyse du lexique de la restauration, les collocations (référentiel restauration).

euh le Saint-Nicolas..._Non c'était le côtes du Rhône..._T'es sûr ? (...)_Pas de nez, pas I 129.odt"> _ Donc, là, on est sur un Côtes du Rhône, $c^{\prime}$ est une appellation génériqu it une appellation générique, hein, " Côtes du Rhône ", pas de domaine particulier, lein, pas de... Appellation générique " Côtes du Rhône ", on est sur un millésime 2010 isi à chaque fois que je donne euh un coup de fouet on voit bien que c'est épaissi. \{ lointé on a plus qu'à l'enfoncer d'un coup sec au marteau. \{S\} Donc là c'est pareil j l, par exemple quand on sert dans une coupe à champagne, voilà, et ben c'est pareil iccord ?\{S\} Donc on a des clients, un couple de clients qui ont l'intention, hein, $\pi$ sont caillés, ils s'égouttent, et au cours de leur affinage on va les laver plusieu par exemple, ou dans le bleu ?\{S\} Au cours de son élaboration, hein dans les roues là ?\{S\} Comme ça ?\{S\} Et ça c'est un couteau entremets ça C'est pas un .... C'est Thi a bouche !_Cul de poule._Ouais voilà cul de poule._(...)_Parce qu'il y a de l'eau d régulier ça très important.\{S\} Et le cul de poule du fait de sa forme arrondie ça $v$ lites attention aussi, à la...à votre cul de poule. $\{S\}$ Qu'il tombe pas directement d en même temps que vous tournez votre cul de poule. \{S\} Donc incorporer délicatement lans un premier temps $j$ 'ai utilisé un cul de poule pourquoi ?\{S\} Parce que je vais $f$ i grammes de farine.\{S\} On utilise un cul de poule, d'accord, ce sont des oeufs enti Ilsion est-ce que je vais utiliser un cul de poule ?\{S\} Je vais utiliser une calotte :l ? _C'est le petit matériel oui._Un cul de poule._Ensuite ?_Cul de poule._Oui._Une : la certitude..._(rires) La bouché ! _cul de poule._Ouais voilà cul de poulé._(...)_ Iriel oui. Un cul de poule. Ensuite ? Cul de poule. Oui. Une balance. D'accord, une

Fig. 7. Extrait de l'analyse du lexique de la restauration, les locutions figées (référentiel restauration).

Pour traiter de manière plus fine le lexique autour de l'eau, le projet de recherche, mené au centre de recherche Grammatica concernant la constitution de ressources linguistiques pour des applications didactiques et linguistiques, adopte une méthodologie qui tient compte de trois types d’unités :

- les unités monolexicales ;

- les unités hybrydes (collocations) ; et

- les unités polylexicales.

En effet, ce projet tient compte du phénomène phraséologique (collocations et locutions) pour le traitement du lexique. Aucun dictionnaire ne distingue de façon systématique ces trois types d'unités.

Du point de vue linguistique et didactique, et dans l'enseignement du FOS dans notre cas, le vocabulaire doit être enseigné en tant qu'unités lexicales. Les unités lexicales prennent en compte les lexèmes mais également les lexies telles que les locutions et les collocations.

Le FOS présente comme particularité, l'étude de lexiques dans des domaines spécialisés. Le lexique recycle les unités existantes (unités monolexicales) pour créer de nouvelles unités lexicales (collocations ou unités polylexicales) qui sont 
utilisées dans les langues de spécialité et deviennent des «termes » mono-référentiels grâce à leur contexte dans le discours. Ces nouvelles unités non seulement enrichissent les langues de spécialité mais également la langue générale en modifiant d'une certaine manière le «sens «global » de la ou des séquence(s) ou « une partie » du sens de la ou séquence(s) (Mangiante et Meneses, 2016). Par exemple, l'unité lexicale « eau » permet de former des collocations :

- eau vive, reste de l'eau mais n’acquiert pas les propriétés humaines « vivant » et « mort » sinon que l’adjectif perd d’une certaine manière son contenu sémantique pour référer à un état de l'eau dans le domaine « maritime » : qui s'agite en permanence et rend la navigation périlleuse.

- $\quad$ eau vive = aguas corrientes

- $\quad$ eau morte = agua estancada

- $\quad$ eau minérale = agua con gas

- $\quad$ eau rougie (mêlée d’un peu de vin rouge).

Domaine de la restauration :

- $\quad$ eau plate $=$ agua del grifo (ES), agua simple (Mex)

- $\quad$ eau minérale $(F R) \rightarrow$ eau pétillante $(F R)=$ agua con gas

- passer sous l'eau froide/chaude

- diluer de l'eau

- $\quad$ verser de l'eau.

Autres :

broc, carafe, cruche, seau d'eau ; pot à eau

Loc. fig., pop. Il y a de l'eau dans le gaz. Il y a quelque chose d'anormal, qui cloche.

\section{Applications didactiques en FOS, l’apport des analyses issues des référentiels de compétences langagières}

L'analyse des enregistrements de dialogues au travail et du lexique de spécialité selon les différentes méthodologies présentées plus haut (lien tâche professionnelle - langue - action, fonctions et régulations linguistiques des genres professionnels, analyse des occurrences lexicales...) permet de concevoir des activités pédagogiques en FOS autour de grilles de compréhension orale mettant en évidence les structures syntaxiques récurrentes et le lexique spécialisé.

Le recours aux corpus numériques peut inciter à des recherches des apprenants, en collaboration avec l'enseignant, pour dégager les occurrences, l'environnement lexical, les collocations... et les relier au protocole actionnel des tâches professionnelles observées dans les référentiels. Les activités doivent permettre une mise ne évidence des protocoles opérationnel et culturel.

En production orale, l'enseignant organisera, dans cette perspective, des simulations de situations orales similaires (filmées) à partir de grilles pré-remplis comportant des informations sur la demande du client, à jouer après un temps de préparation par groupe.

La correction collective s'effectuera à partir des enregistrements vidéo réalisés et le visionnage de la situation issue du corpus.

Exemples d'activités en ligne issues des référentiels : 


\section{Texte à trous}

Correction Recommencer

?

Complétez par les mots ci-dessous :

l'eau - plonge - plateaux - eau - poubelle -

plantes - l'évier - l'office - sac poubelle -

fond - jettes - fleurs -

Jonc ce qu'il va falloir faire, c'est éventuellement mettre sur les d'accord? Al' , on verifiera si on a bien un , d'accord, et tu vas les ramener directement à l' Jas, elles sont toutes fanées et tout mettre à la , comme ça, tu vides tout, hein, toutes les on ne les garde Ça je jette tout?

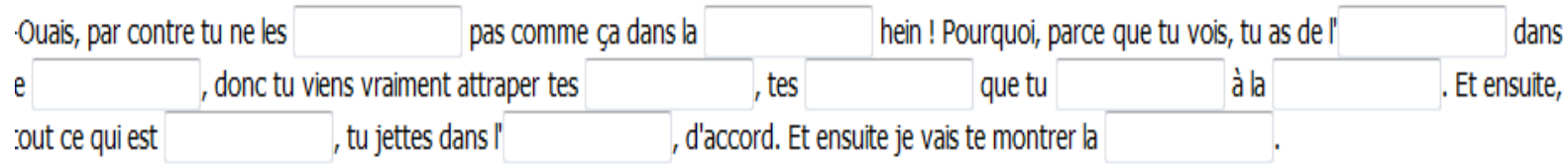

Fig. 7. Texte à trous à partir de la transcription d'un enregistrement issu du référentiel restauration.

\section{Les produits d'entretien du linge}

\section{Correction Recommencer}

\section{(2) La Buanderie}

Les produits d'entretien du linge

Faites glisser la bonne définition sous le mot correspondant.

\begin{tabular}{|c|}
\hline $\begin{array}{l}\text { Il abîme le linge et les machines à laver. On } \\
\text { utilise alors ce produit pour ôter le calcaire } \\
\text { d'une machine à laver. }\end{array}$ \\
\hline C'est un produit qui sert à laver le linge \\
\hline $\begin{array}{l}\text { Produit qui permet d'enlever les taches sur les } \\
\text { textiles. }\end{array}$ \\
\hline $\begin{array}{l}\text { C'est un produit de rinçage destiné à assouplir } \\
\text { le linge après son lavage. (Synonyme: } \\
\text { Adoucissant) }\end{array}$ \\
\hline
\end{tabular}
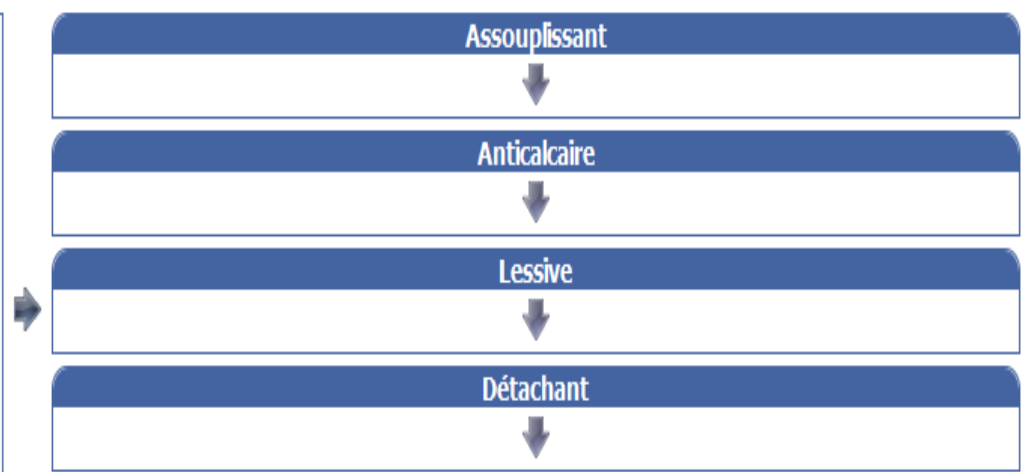

Fig. 8. Exercice de classement du lexique issu du référentiel hôtellerie. 


\section{En conclusion}

L'analyse discursive et lexicale des corpus professionnels extraits des référentiels et l'étude des enregistrements des dialogues en situations de travail conduisent à des applications didactiques contextualisées destinées à des publics de travailleurs migrants désireux de s’insérer dans le monde du travail francophone, par exemple.

L'étude des discours et du lexique spécialisé révèle ainsi les liens qui unissent la langue à l'action au sein des tâches professionnelles à l'œuvre dans les extraits étudiés.

La démarche didactique de conception de programmes de FOS intègre une étape d'analyse des données collectées sur le terrain de la pratique langagière. Il est clair que le recours à des corpus existants peut compléter utilement ces données dont la collecte n'est pas toujours réalisable. Complétés par des outils d'analyse de discours, ces corpus s'avèrent des supports de cours intéressants mais nécessitent une contextualisation qui est en partie assurée par leur intégration à des référentiels de compétences langagières en milieu professionnel.

Ces référentiels et les outils d'analyse associés font apparaître les liens qui unissent la langue et l'action au travail au sein des tâches professionnelles observées, liens qui relèvent des protocoles actionnels et socioculturels qui régissent les activités professionnelles (Mangiante et Meneses, 2016).

«L'actualisation de ce que l'on sait dans un contexte singulier (marqué par les relations de travail, une culture institutionnelle, des aléas, des contraintes temporelles, des ressources ...) est révélatrice du « passage » à la compétence. Celle-ci se réalise dans l'action. Elle ne lui préexiste pas. [...] Il n'y a de compétence que de compétence en acte. » (Le Boterf, 2000).

\section{Références bibliographiques}

BEACCO, Jean-Claude (2004). « Trois perspectives linguistiques sur la notion de genre discursif », Langages, $n^{\circ} 153$, vol. 38.

BouTET, Josian (2001). «La part langagière du travail : bilan et évolution », Langage et Société n98, Editions de la maison des sciences de l'homme, p. 17-42.

Boutet, Josiane et Maingueneau, Dominique (2005), "Sociolinguistique et analyse de discours : façons de dire, façons de faire », Langage et société 4/2005 ( $\left.\mathrm{n}^{\circ} 114\right)$, p. 15-47.

BronCKART, Jean-Paul (2004). Agir et discours en situations de travail. Chapitre 5 : « Présentation de la recherche ». Cahiers de la section des sciences de l'éducation n¹03. Université de Genève, p. 119 - 144.

GREEN, Bill (1997). Literacy information and the learning society. Joint conference of the Australian Association for the teaching of English, the Australian Literacy Educators’ Association and the Australian School Library Association, Darwin.

Grünhage-Monetti, Matilde ; Halewwijn, Elwine et Holland, Chris (2003). Odysseus - La langue seconde sur le lieu de travail. Strasbourg : Conseil de l'Europe.

HABERT, Benoît (2005). Instruments et ressources électroniques pour le français. Gap/Paris : Ophrys, 170 p.

HADJi, Charles (2000), L'évaluation, règles du jeu. Paris : ESF éditeur.

Kerbrat-Oreccihioni, Catherine (2012). «Le contexte revisité ». Corela [En ligne], HS-11 | 2012, mis en ligne le 02 avril 2012 <http://corela.revues.org/2627> [Consulté le 31 mai 2015].

Le BOTERF, Guy (2000). Construire les compétences individuelles et collectives. Paris, Editions d'organisation.

MANGIANTE, Jean-Marc (2011). L’intégration linguistique des migrants. Etat des lieux et perspectives. Arras : APU, p. 107-122.

Mangiante, Jean-Marc et Meneses, Luis (2016), «L’Analyse de données en FOS : caractéristiques méthodologiques et outils numériques », Points Communs - REDLOS n³, Paris : CCIP - IDF.

MAngiante, Jean-Marc et PARPETte, Chantal (2004), Le Français sur Objectif Spécifique, Paris : Hachette FLE.

MANGIANTE, Jean-Marc et PARPETTE, Chantal (2011), Le Français sur Objectif Universitaire, Grenoble : PUG.

MOURLHON-DALlies, Florence (2008). Enseigner une langue à des fins professionnelles. Paris : Didier.

RicheR, Jean-Jacques (2008). Le français sur objectifs spécifiques: une didactique spécialisée? dans Synergies Chine $n^{\circ} 3$, GERFLINT, p. 15-30.

Normand, Victor (1992). Task Modelling in HCI : Purposes and Means, State of the Art and Research Issues, Rapport de Recherche, Bagneux : Thomson-CSF, Division SDC. 